\title{
Roles of the MYST Family in the Pathogenesis of Alzheimer's Disease via Histone or Non-histone Acetylation
}

\author{
Yuhong Li' ${ }^{1,2}$, Hui Huang1, Man Zhu', Hua Bai', 3, Xiaowei Huang1,* \\ ${ }^{1}$ State Key Lab for Conservation and Utilization of Bio-Resources, Yunnan University, Kunming, Yunnan, China. \\ ${ }^{2}$ Yunnan Institute of Tropical Crops, Jinghong, China \\ ${ }^{3}$ College of Public Health, Kunming Medical University, Kunming, China
}

[Received January 26, 2020; Revised March 27, 2020; Accepted March 29, 2020]

\begin{abstract}
Alzheimer's disease (AD) is one of the most common neurodegenerative diseases and a major cause of death among elderly individuals. The etiology of AD involves a combination of genetic, environmental, and lifestyle factors. A number of epigenetic alterations in AD have recently been reported; for example, studies have found an increase in histone acetylation in patients with AD and the protective function of histone deacetylase inhibitors. The histone acetylases in the MYST family are involved in a number of key nuclear processes, such as gene-specific transcriptional regulation, DNA replication, and DNA damage response. Therefore, it is not surprising that they contribute to epigenetic regulation as an intermediary between genetic and environmental factors. MYST proteins also exert acetylation activity on non-histone proteins that are closely associated with the pathogenesis of AD. In this review, we summarized the current understanding of the roles of MYST acetyltransferases in physiological functions and pathological processes related to AD. Additionally, using published RNA-seq, ChIP-seq, and ChIP-chip data, we identified enriched pathways to further evaluate the correlation between MYST and AD. The recent research described in this review supports the importance of epigenetic modifications and the MYST family in AD, providing a basis for future functional studies.
\end{abstract}

Key words: Alzheimer's disease, MYST, Epigenetic, MOZ, MORF, HBO1, hMOF

\section{Alzheimer's disease}

Alzheimer's disease (AD) was first discovered in 1906 and is now considered the main cause of dementia, making it a major issue in the context of the acceleration of aging in the population. As a primary degenerative encephalopathy, its clinical manifestations include a decline in the memory functions in the early stage and a gradual, complete loss of cognitive ability, speech, and even a normal behavioral function. Since the disease course has been thought to be irreversible, $\mathrm{AD}$ is an intractable medical problem and a major social burden.
Since the year 2000, the mortality rate for AD has increased by $89 \%$ while that for heart diseases has decreased by $14 \%$ during the same period. As this trend continues, it is estimated that the total medical investment in $\mathrm{AD}$ may reach 1.1 trillion dollars by the year 2050 in the United States alone [1].

The characteristic pathological changes in most patients with $\mathrm{AD}$ are consistent and include $\beta$-amyloid deposition, entanglement of the neurogenic fibers, senile plaque formation, reduction in the number of memory neurons, and atrophy of the cerebral cortex. However, the etiology involves a combination of genetic,

*Correspondence should be addressed to: Dr. Xiaowei Huang, State Key Lab for Conservation and Utilization of Bio-Resources, Yunnan University, Kunming 650091, Yunnan, China. Email: xwhuang@ynu.edu.cn.

Copyright: () $2020 \mathrm{Li} \mathrm{Y}$ et al. This is an open-access article distributed under the terms of the Creative Commons Attribution License, which permits unrestricted use, distribution, and reproduction in any medium, provided the original author and source are credited. 
environmental, and lifestyle factors. At the genetic level, mutations in genes encoding the amyloid precursor protein (APP) and presenilins 1 and 2 have been detected in the autosomal dominant form of AD. However, autosomal dominance accounts for only a small proportion (about $5 \%$ ) of cases. Among sporadic AD, the $\varepsilon 4$ allele of $A P O E$ is the greatest risk factor, responsible for about $30 \%-35 \%$ of cases [2]. Various environmental inputs, such as pesticide exposure, a high-fat diet, and a sedentary lifestyle, have been hypothesized to remodel the epigenome and thus are closely related to the occurrence of AD. For example, the incidence of degeneration of the frontotemporal lobe increases with age along with an excessive production of ROS and decreased cellular antioxidant defenses [2]. ROS accumulation can alter DNA methylation and histone acetylation, thereby influencing the amyloid- $\beta$ production [3]. Accordingly, epigenetic changes in the expression of pathogenic genes have been suggested to be another major determinant of AD development [4].

\section{Histone acetylases}

Histone acetylation, one of the main types of histone modification, involves the addition of an acetyl group to lysine residues in the N-terminal tail or on the surface of the nucleosome core of histones. Acetylated histones usually relax the chromatin structure, thereby increasing transcription. Acetylation also affects the replication and repair of DNA [5]. This modification is commonly performed by histone acetyltransferases (HATs) at specific lysine sites of histones $\mathrm{H} 2 \mathrm{~A}, \mathrm{H} 2 \mathrm{~B}, \mathrm{H} 3$, and $\mathrm{H} 4$. HATs have been identified in a variety of eukaryotic organisms from yeast to humans and are classified into three primary subclasses according to a structural homology: (1) the GNAT (Gcn5-related $N$-acetylase) family, containing Gnc5, PCAF, and the elongator complex protein 3 (ELP3); (2) the MYST family, consisting of Tip60, MOZ/MYST3, MORF/MYST4, HBO1/MYST2, and hMOF/MYST1; and (3) the p300/CBP (CREB-binding protein) family, including p300 and CBP [6].

Direct links between HATs and AD have been established; there is an evidence of the increased histone acetylation in $\mathrm{AD}$ and protective functions of the histone deacetylase inhibitors [7]. In particular, the roles of the GNAT and p300/CBP families in AD have been demonstrated. $\mathrm{H} 2 \mathrm{~B}$ and $\mathrm{H} 4$ acetylation in the rat dorsal hippocampus produced by PCAF, CBP, and p300 may enhance the expression of memory/plasticity-related genes and therefore be helpful in consolidating a spatial memory. However, PCAF selectively acetylates NF- $\mathrm{kB}$ at Lys-122, which triggers the inflammatory response induced by $A \beta$ in mammalian cells $[8,9]$. The p300/CBP family exhibits a neuroprotective activity in the context of $\mathrm{AD}$ development, and therefore, loss or over-expression of $\mathrm{CBP} / \mathrm{p} 300$ contributes to the neuronal death $[10,11]$. In addition to a p300-mediated histone acetylation, the acetyltransferase activity of p300 influences the hyperacetylation of the tau proteins in AD brains [12].

In addition to the direct roles of HATs, there is an evidence of additional indirect effects in AD. For example, GCN5 acetylates CDK5 at Lys33, which affects the kinase activity [13]. Cdk5 activation is responsible for abnormalities of the amyloid, tau, and neurofilament proteins in AD, implying that GCN5 may be critically involved in the pathogenesis of $\mathrm{AD}$, but additional experimental evidence is needed $[14,15,16]$.

Here, we reviewed recent evidence of the key roles of epigenetic mechanisms in $\mathrm{AD}$ with a focus on the MYST family. Furthermore, we utilized publicly available data from high-throughput genomic assays to clarify the mechanisms by which the MYST proteins contribute to the pathogenesis of the disease.

\section{The MYST family and AD}

The MYST family currently includes five human HATs, Tip60, MOZ, MORF, HBO1, and MOF, and orthologs have been detected in other eukaryotes. They function in multi-subunit protein complexes with evolutionarily conserved compositions. With respect to the structure, MYST acetyltransferases have a distinct histone acetyltransferase domain, which contains a $\mathrm{C} 2 \mathrm{HC}$ zinc finger and an acetyl-CoA-binding site [17]. Through a MYST-dependent chromatin acetylation, the MYST family regulates a variety of cellular processes, ranging from gene regulation to DNA repair, cell cycle, stem cell homeostasis, and development. Non-chromatin substrates of MYST enzymes and a few cellular pathways independent of a MYST-mediated histone acetylation have recently been reported, emphasizing the multifunctional nature of this highly conserved acetyltransferase family. Different types of MYST proteins have distinct physiological functions and roles in $\mathrm{AD}$ as summarized below.

\section{Tip60 (KAT5)}

TIP60 (Tat-interactive protein 60) functions as a key regulator of homeostasis via the regulation of gene transcription, DNA repair, and apoptosis with target genes such as the ATM protein kinase and p53 tumor suppressor [18]. Its roles in $A D$ have been investigated most intensively among members of the MYST family and have been summarized in Figure 1 [19]. Tip60 directly affects the amyloid- $\beta$ precursor protein (APP) and the tau protein. In the pathological changes of AD, Fe65 (a ligand 
of APP) binds to the intracellular subdomain of APP (AICD), and their interaction in the nervous system significantly increases the transfer of APP to the cell surface as well as the accumulation of $\beta$-amyloid peptides in vitro [20]. However, a recent study has shown that Tip60 forms a multimeric complex with Fe65 and AICD in the cytoplasm, thereby stimulating gene transcription via recruitment of a Tip60-dependent histone acetylation [21, 22]. Studies of cells co-expressing AICD/FE65/TIP60 have also shown that the expression of AICD as well as the complex downregulates Stathmin1, a cytoskeleton-associated protein, and therefore disrupts microtubule dynamics and influences the neurofibrillary tangles in $\mathrm{AD}[23,24]$. In addition, the co-localization and binding of Fe65 and the tau protein in neurofibrillary tangles of the hippocampus suggest that Fe65 plays an additional role in the crosslinking between the $\beta$-amyloid peptides and neurofibrillary tangles in $\mathrm{AD}[25,26]$. However, the involvement of Tip60 in the abovementioned pathological process requires further elucidation.

Studies of Tip60 have provided substantial insights into cognitive disorders and epigenetics. Experimental data generated by $\mathrm{Xu}$ and colleagues have shown that a steady cognitive deterioration and the corresponding

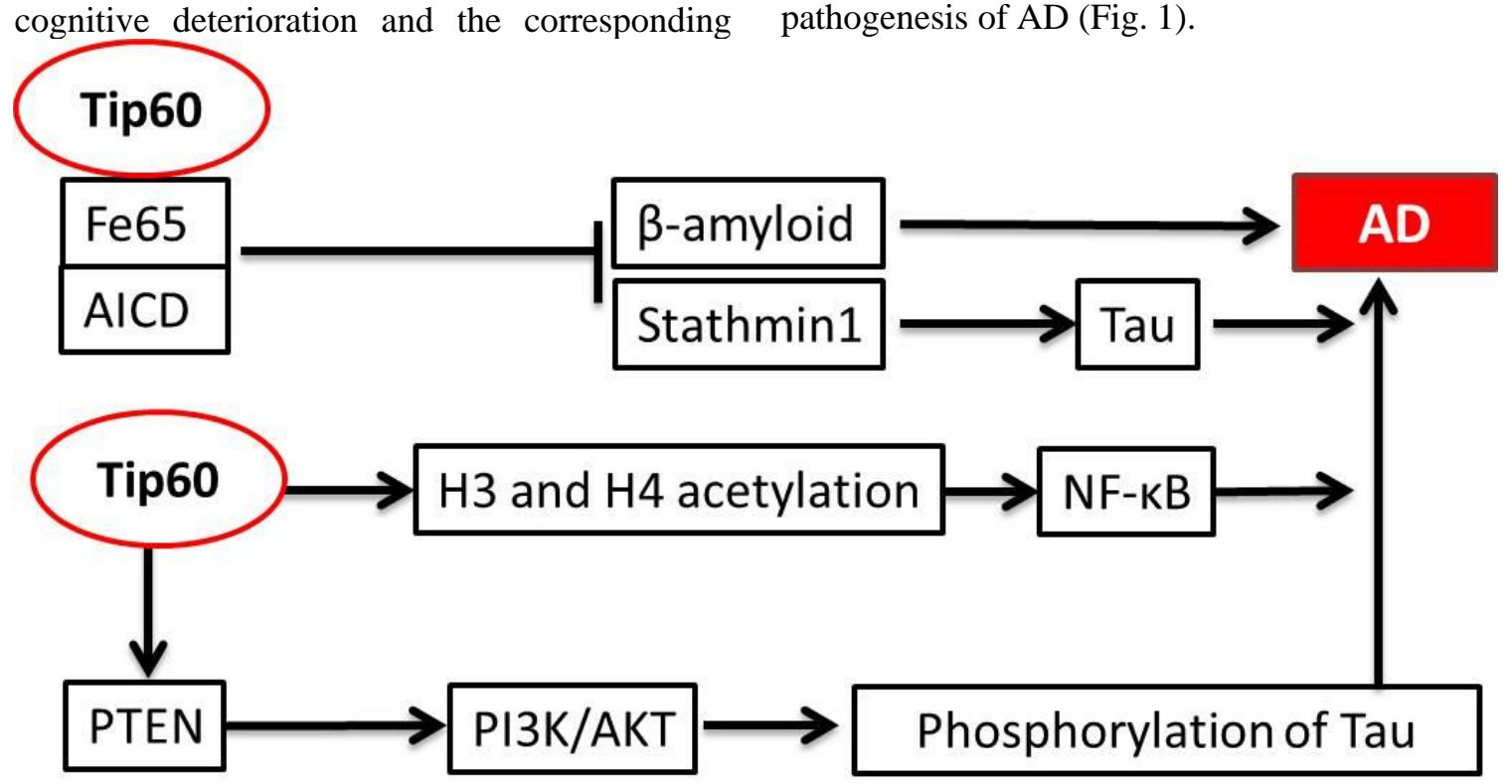

Figure 1. Summarized diagram of Tip60 in AD. First, the complex composed of Fe65, AICD, and Tip60 can inhibit $\beta$-amyloid and Stathmin1 while Stathmin1 can promote tau phosphorylation. At the same time, Tip60 directly acetylates the H3/H4 histones and activates PTEN, thereby affecting the inflammatory pathogenesis of AD and phosphorylation of tau, respectively.

Using ChIP-seq data for Tip60 (GSE76612) and RNA-seq data for the Tip60-related genes generated by $\mathrm{Xu}$ and colleagues, we performed a PANTHER pathway analysis (http://pantherdb.org/; http://kobas.cbi.pku. edu.cn/anno_iden.php) and identified enrichment for the accumulation of $\beta$-amyloid peptides are effectively blocked or even rescued by increasing the Tip60 levels in the mushroom body of Drosophila [27]. To determine the molecular mechanisms underlying the neuroprotective function of Tip60, ChIP-seq analysis has identified a variety of cognitive genes that are dysregulated in Tip60mutant flies [28]. Additionally, environmental enrichment has been hypothesized to improve the cognitive ability in neuropathic diseases, and Tip60 may mediate these beneficial effects [29].

In addition to its direct interactions with APP, thereby improving the cognitive ability and conferring neuroprotection, Tip60 may be involved in the regulation of other AD-related biological processes. For example, Tip60 positively modulates the PTEN levels and subsequently activates PI3K/AKT in cholangiocarcinoma tissues [30, 31]. However, an earlier report showed that AKT contributed to the phosphorylation of tau at Thr212 and Ser214 and was a risk factor for AD [30]. Additionally, Tip60 directly catalyzes H3 and H4 acetylation in the promoter regions of the NF- $\kappa \mathrm{B}$ target genes (e.g., IL-6, IL-8, XIAP, and $c I A P$ ) and maintains levels of acetylated Lys-310 of RelA/p65 [32]. NF- $\kappa \mathrm{B}$ is also widely appreciated as a key gene in the inflammatory pathogenesis of AD (Fig. 1). 


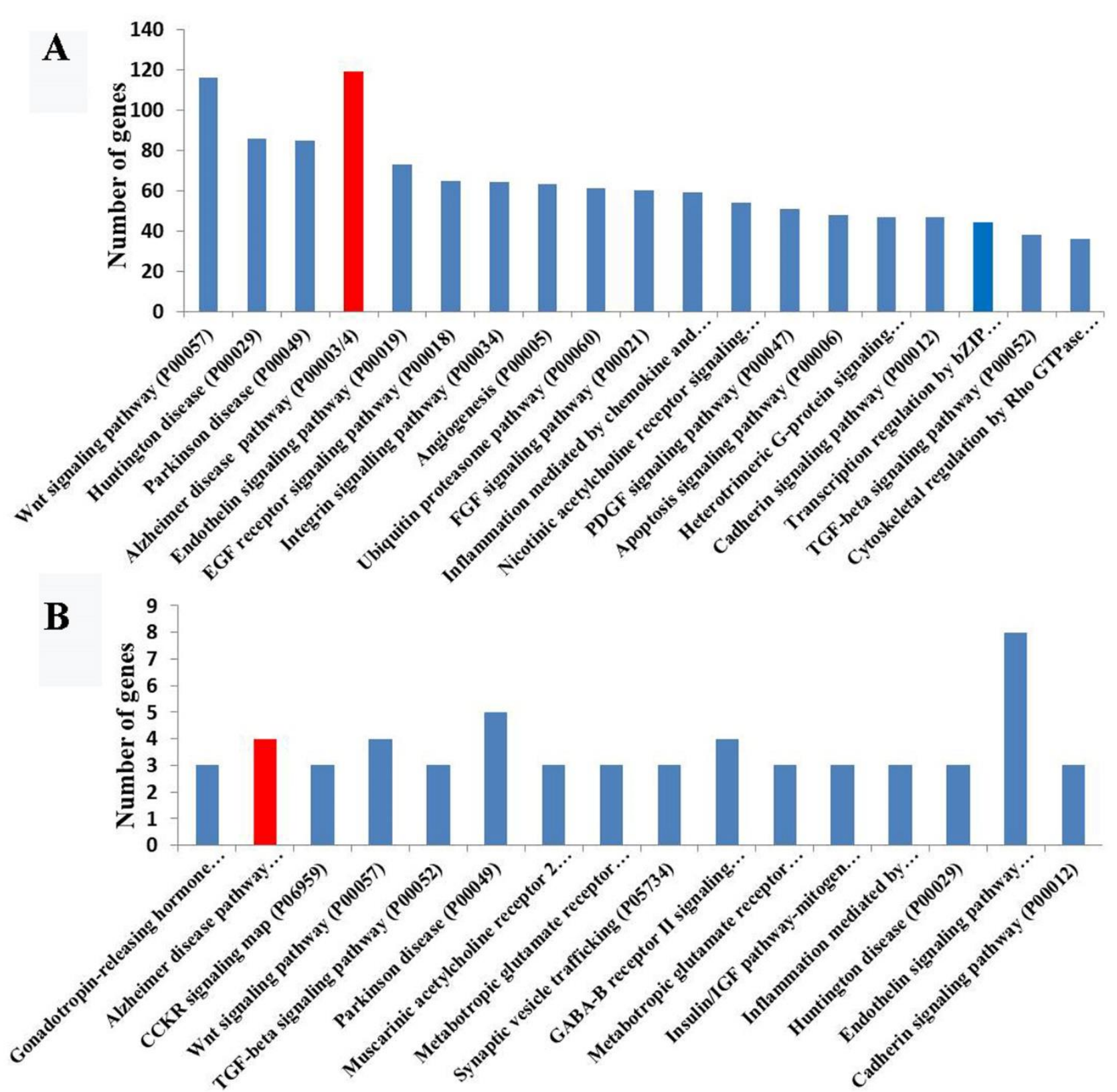

Figure 2. Tip60-mediated target genes involved in AD. PANTHER pathway analyses of the differentially expressed genes determined by ChIP-seq using a Tip60 antibody in Drosophila melanogaster (A) and determined by RNA-seq in Tip60-mutant Drosophila melanogaster (B). The 20 most highly enriched pathway entries have been displayed.

\section{MOZ/MYST-3 (KAT6A) AND MORF/MYST4 (KAT6B)}

KAT6A, also known as MOZ (monocytic leukemia zinc finger protein) or MYST3, has crucial functions in the long-term reproduction of hematopoietic stem cells and the formation of memory B cells [33]. Although MOZ seems to indirectly affect $\mathrm{AD}$, a few studies have shown that it is involved in the self-renewal of neural stem cells
(NSCs) and is a potential therapeutic target for AD [34, $35,36]$. MOZ can silence the $\mathrm{p} 16^{\mathrm{INK} 4 \mathrm{a}}$ expression by binding to the promoter of $\mathrm{p} 16^{\mathrm{INK} 4 \mathrm{a}}$, which is critical for the self-renewal and proliferation of NSCs [37]. Increased neuronal proliferation may lead to the attenuation of an $\mathrm{A} \beta$-induced neurodegeneration in an $\mathrm{AD}$ mouse model [38]. Furthermore, the zinc finger and acetyltransferase domains of MOZ fuse to the gene encoding the E1Aassociated protein $\mathrm{p} 300(\mathrm{p} 300)$ at $\mathrm{t}(8 ; 22)(\mathrm{p} 11 ; \mathrm{q} 13)$ in 
acute monocytic leukemia based on FISH experiments $[39,40]$. The role of $\mathrm{p} 300$ in $\mathrm{AD}$ is supported by an experimental evidence that the addition of a p300 inhibitor significantly suppresses the expression of presenilin 1 (PS1) and the beta-site amyloid precursor protein cleaving enzyme 1 (BACE1) via a reduced $\mathrm{H} 3$ acetylation within their promoter regions [41, 42]. In addition, p300 has an effect on the p300-mediated acetylation of tau in a concentration-dependent manner and in turn represses the tau assembly [43, 44], suggesting that $\mathrm{MOZ} / \mathrm{p} 300$ influences the aggregation of tau (Fig. 3).

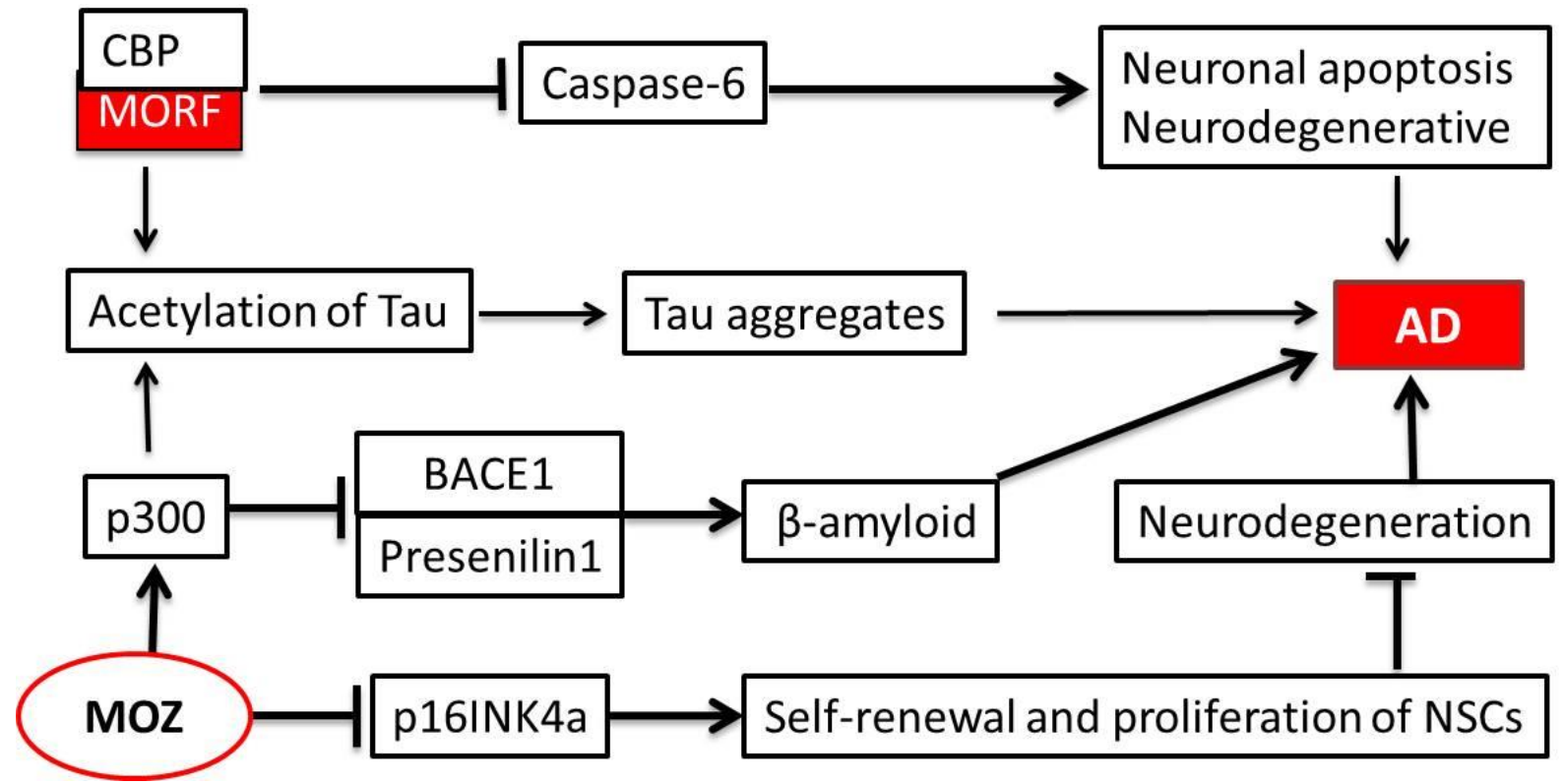

Figure 3. Involvement of MOZ and MORF in AD. MOZ binds to the promoter of p16INK4a and silences the p16INK4a expression, which in turn inhibits the self-renewal and proliferation of NSCs. The interaction of MOZ/p300 can acetylate tau or suppress the expression of Presenilin1 and BACE1. Additionally, the fusion genes of MORF and CBP can aggravate the pathogenesis of $\mathrm{AD}$ by either inhibiting the expression of the neuronal apoptosis factor (caspase-6) or promoting the abnormal accumulation of acetylated tau.

MORF/MYST4 (monocytic leukemia zinc finger protein-related factor), also known as KAT6B, is highly homologous to MOZ/MYST3 (KAT6A) and preferentially acetylates $\mathrm{H} 2 \mathrm{~A}, \mathrm{H} 3$, and $\mathrm{H} 4$ in vitro [45]. Interestingly, MORF/MYST4 (KAT6B) is also involved in acute myeloid leukemia and the myelodysplastic syndrome with fusion to the CREB-binding protein (CBP) instead of $\mathrm{p} 300$, similar to that exhibited by MYST3/KAT6A [46]. In fact, CBP and p300 are highly homologous (with an overall identity of $>70 \%$ ) and have highly overlapping functions via their interactions with similar partners and substrates [47]. Similar to the HATs described above, CBP/p300 in the neural system can confer neuroprotective functions and their aberrant expression leads to neuronal apoptosis and neurodegenerative diseases via a caspase- 6 activation [10, $11,47]$. The acetylation of tau at lys 28 via $\mathrm{CBP} / \mathrm{p} 300$ impairs the interaction between tau and microtubules, contributing to the formation of insoluble tau aggregates in $\mathrm{AD}$ [48] (Figure 3). However, the involvement of MOZ/MYST3 (KAT6A) or MORF/MYST4 (KAT6B) in the effects of $\mathrm{CBP} / \mathrm{p} 300$ remains unclear.
We also performed a PANTHER pathway analysis using RNA-seq data of the MOZ-TIF2 fusion gene in leukemic stem cells (Figure 4A), MOZ knockdown in SUM-52 cells (Figure 4B), and MORF knockdown in HEK293 and HeLa cells (Figure 4C). Our results illustrated enrichment for the AD-presenilin pathway (P00004) and the AD-amyloid secretase pathway (P00003) in all datasets.

\section{HBO1/MYST-2 (KAT7)}

HBO1 (histone acetyltransferase binding to ORC1) interacts with ING4 and BRPF3 to acetylate $\mathrm{H} 4$ at lysines $5,8,12$, and 14 , thereby activating gene transcription. HBO1 also functions as a co-activator of the DNA prereplication complex formation based on a genome-wide analysis showing high enrichment for HBO1, BRPF3, and $\mathrm{H} 3 \mathrm{~K} 14 \mathrm{ac}$ at ORC1-binding sites and replication origins [49]. A few indirect correlations between $\mathrm{HBO} 1$ and $\mathrm{AD}$ have been inferred. For example, HBO1 increases the activity of $\beta$-catenin in the Wnt signaling pathway in both the J82 and T24 cells [50], and either a reduced $\beta$-catenin 
in $\mathrm{AD}$ or the Wnt inhibitor Dkk1 (Dickkopf-1) positively regulates tau phosphorylation and exacerbates the cognitive deficits, establishing a link between HBO1 and $\mathrm{AD}[51,52]$.

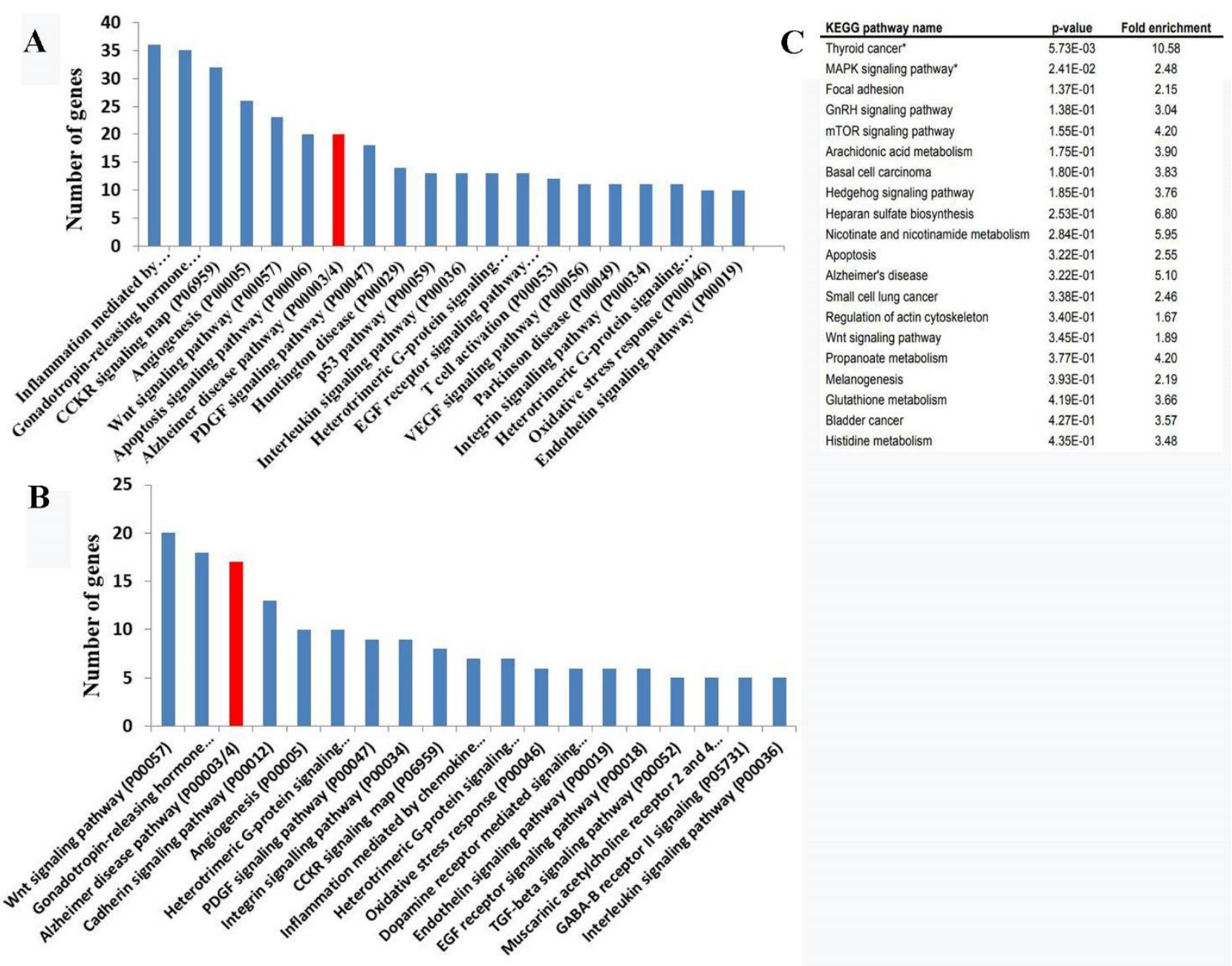

Figure 4. MOZ-mediated target genes involved in AD. PANTHER pathway analyses of the differentially expressed genes in leukemic stem cells with the MOZ-TIF2 fusion gene (A), MOZ-knockdown in SUM-52 cells (B), and siRNA knockdown of MORF in the HEK 293 and HeLa cells (C). The 20 most highly enriched pathway entries have been displayed.

To determine the roles of HBO1 in AD, ChIP-seq and ChIP-chip data for human cell lines were analyzed to identify target genes that bind to HBO1 $[49,53]$. The PANTHER pathway (Figures 5A and 5B) and pathway enrichment analyses (Figure 5C) showed that targets of HBO1 included genes associated with neurodegenerative diseases, such as AD, HD (Huntington's disease), and PD (Parkinson's disease).

\section{MOF/MYST1 (KAT8)}

MOF (males absent on the first) usually functions as a catalytic subunit in acetyltransferase complexes. In human cells, it can form two different protein complexes (MSL and NSL), both of which acetylate histone $\mathrm{H} 4$ at lysine 16 (H4K16). However, the NSL complex has wider substrate specificities with histone $\mathrm{H} 4$ acetylated at lysine 5 and 8 (H4K5 and H4K8) [54, 55]. Functional studies have evaluated the direct correlation between the MOF complex and the pathogenesis of $\mathrm{AD}$, revealing that the complex regulates a series of cellular processes or pathways that participate in the development of $\mathrm{AD}$. There is substantial evidence that the accumulation of reactive oxygen species (ROS), deleterious effects on biomolecules (e.g., proteins, DNA/RNA, and lipids) from an oxidative stress, mitochondrial dysfunction directly or indirectly caused by ROS, and an eventual apoptosis or necrosis of neurons contribute to the etiology of AD [56, 57]. Silencing or overexpression of MOF may downregulate the ROS-generating gene Nox4 or enhance catalase and MnSOD dependent on the activity of MOF histone acetyltransferase and the level of H4K16, 
respectively [58]. Consistent with these findings, RNAseq data for the MOF deficiency mutant showed a significant downregulation of antioxidant genes, leading to an increased production of ROS [59]. As a dualtranscriptional regulator of the nuclear and the mitochondrial genomes connecting epigenetics and metabolism, MOF knockout leads to a mitochondrial degeneration, disturbance of the mitochondrial nutrient metabolism, and deregulation of the oxidative phosphorylation pathways [60]. Lysosomes also play an important role in $\mathrm{AD}$. On one hand, $\mathrm{A} \beta$ impairs the lysosomal function via lysosomal de-acidification and membrane permeabilization. On the other hand, the levels of the lysosomal proteins, such as LAMP1 (lysosomeassociated membrane protein 1) and LAMP2, the important two markers of lysosomal function, change with the occurrence of $\mathrm{A} \beta$ deposition or $\mathrm{AD}[61,62]$. A genome-wide expression analysis further showed that the histone acetyltransferase MOF was able to maintain the transcription of the lysosome-related genes [63] (Figure $6)$.

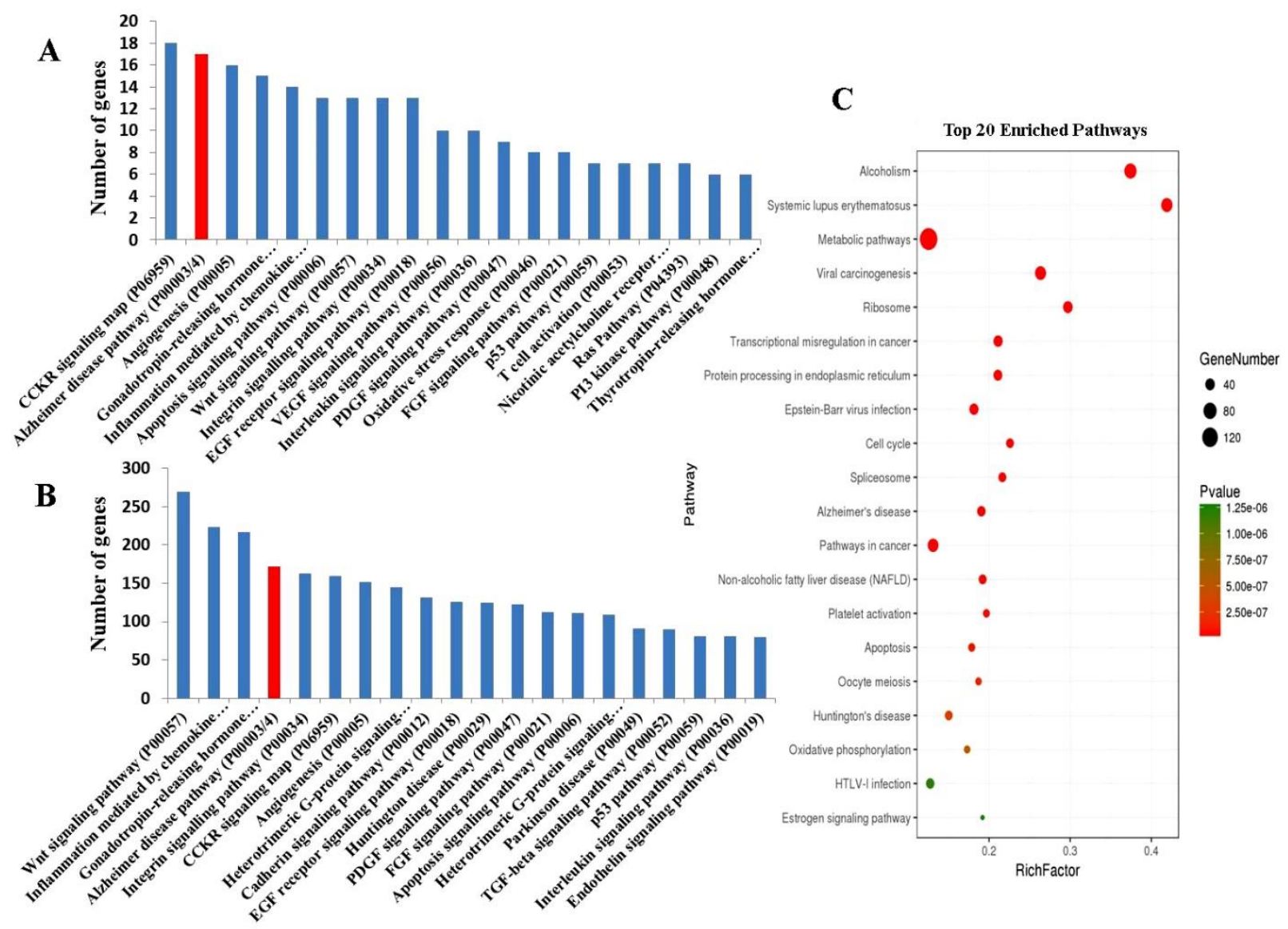

Figure 5. HBO1-mediated target genes involved in AD. PANTHER pathway analyses based on ChIP-seq data obtained using an HBO1 antibody (A), ChIP-chip data using HBO1 antibodies in K562 cells (B), and a pathway enrichment analysis of ChIPchip data for K562 cells (C). The 20 most highly enriched pathway entries have been displayed.

To establish the direct link between MOF and AD, we performed an additional PANTHER pathway analysis based on ChIP-seq data for MOF-binding motifs in the embryonic stem cells [64] (Figure 7A) and PANTHER pathway analyses of the genes that were significantly altered in the MOF-deleted mouse embryonic fibroblasts [63] (Figure 7B). Various genes associated with the pathogenesis of $\mathrm{AD}$ as well as the $\mathrm{AD}$-related cellular processes were identified. 


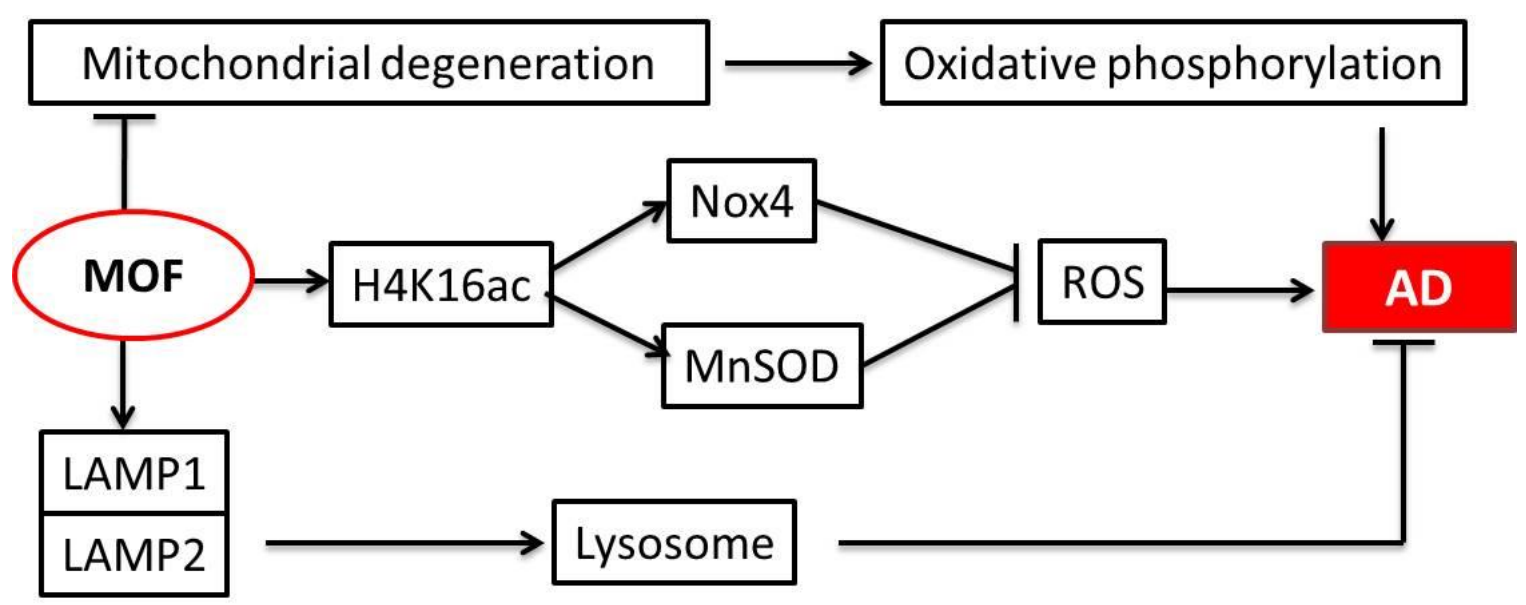

Figure 6. The roles of MOF in AD. MOF can inhibit the degeneration of mitochondria and decrease AD induced by an oxidative phosphorylation. Acetylating H4K16 and MOF also activates Nox4 and MnSOD as well as decreases ROS. Finally, MOF activates lysosomes via LAMP1 and LAMP2.
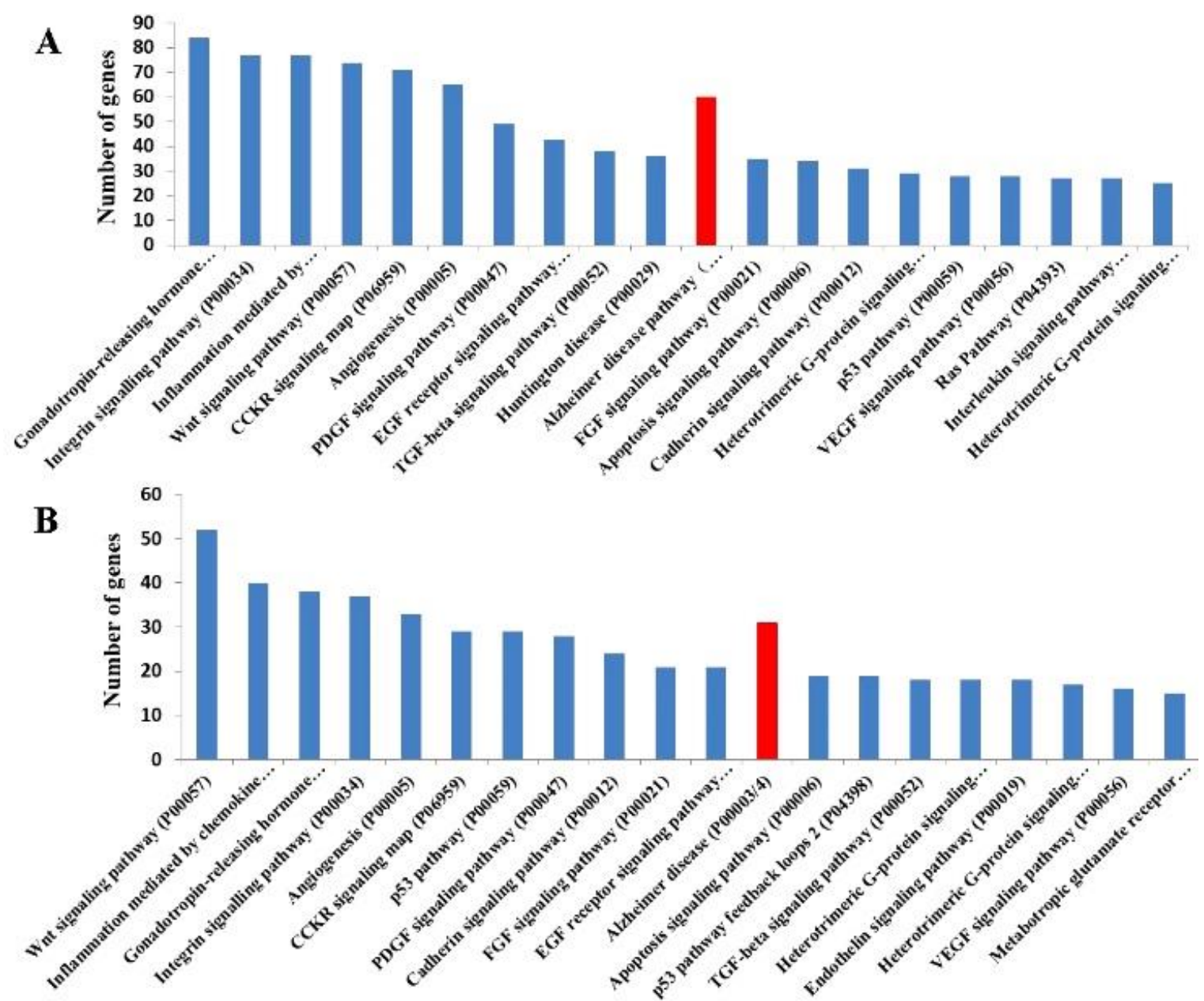

Figure 7. MOF-mediated target genes involved in AD. PANTHER pathway analysis of ChIP-seq data using an MOF antibody in the embryonic stem cells (A), and genes that were significantly altered in the MOF-deleted mouse embryonic fibroblasts (B). The 20 most highly enriched pathway entries have been displayed. 


\section{Conclusions}

Studies of the relationships between HATs and neurodegenerative diseases have revealed that the MYST family has direct effects on AD, PD, and HD. However, partial relationships among them are mediated by intermediate cellular processes or genes. For example, the Wnt signaling plays critical roles in several cellular processes, including synaptic activity, pre- and postsynaptic assembly, function at the glutamatergic synapses, neurogenesis, and neuroprotective memory consolidation. Furthermore, TGF- $\beta$, apoptosis, and/or oxidative stress response pathways with well-established roles in AD are targets of MYST. Our analysis of publicly available high-throughput data supports these previous findings and provides additional insight into the mechanisms by which this protein family contributes to AD. Collectively, additional experimental studies of targets of TIP60, MOZ, HBO1, and MOF as well as direct and indirect contributors to $\mathrm{AD}$ will be needed to validate their roles.

\section{Acknowledgements}

This research was supported by the State Key Lab for Conservation and Utilization of Bio-Resources, Yunnan Institute of Tropical Crops and College of Public Health, Kunming Medical University, and the Yunnan Provincial Science and Technology Department (2019FA046).

\section{Competing interests}

The authors declare that they have no competing interests.

\section{References}

[1] Alzheimer's Association (2018). Alzheimer's disease facts and figures. Alzheimers. Dement 14: 367-429.

[2] Maloney B and Lahiri DK et al. (2016). Epigenetics of dementia: understanding the disease as a transformation rather than a state. Lancet Neurol, 15: 760-774.

[3] Zuo L, Benjamin T. Hemmelgarn, Chia-Chen Chuang et al. (2015). The Role of Oxidative Stress-Induced Epigenetic Alterations in Amyloid- $\beta$ Production in Alzheimer's disease. Oxid Med Cell Longev, 13:604658.

[4] Sanchez-Mut, JV and Graff J (2015). Epigenetic Alterations in Alzheimer's Disease. Front Behav Neurosci, 9: 347.

[5] Phillips DMP (1963). The presence of acetyl groups of histones. Biochem J, 87: 258-263.

[6] Roth SY, Denu JM., and Allis CD (2001). Histone acetyltransferases. Annu. Rev. Biochem, 70: 81-120.
Lu X, Wang L, Yu C et al. (2015). Histone acetylation modifiers in the pathogenesis of Alzheimer's disease. Front Cell Neurosci, 9: 226.

Park SY, Lee YH, Seong AR et al. (2013). Selective inhibition of PCAF suppresses microglial-mediated $\beta$ amyloid neurotoxicity. Int J Mol Med, 32: 469-475.

Park SY, Kim MJ, Kim YJ et al. (2015). Selective PCAF inhibitor ameliorates cognitive and behavioral deficits by suppressing NF- $\mathrm{B}$ mediated neuro inflammation induced by $A \beta$ in a model of Alzheimer's disease. Int J Mol Med, 35:1109-1118.

Rouaux C, Jokic N, Mbebi C et al. (2003). Critical loss of $\mathrm{CBP} / \mathrm{p} 300$ histone acetylase activity by caspase-6 during neurodegeneration. EMBO J, 22:6537-6549.

Rouaux C, Loeffler JP, Boutillier AL et al. (2004). Targeting CREB-binding protein (CBP) loss of function as a therapeutic strategy in neurological disorders. Biochem Pharmacol, 68:1157-1164.

Aubry S, Shin W, Crary JF et al. (2015). Assembly and interrogation of Alzheimer's disease genetic networks reveal novel regulators of progression. PLoS ONE, e0120352.

Lee J, Yun N, Kim C et al. (2014). Acetylation of cyclin-dependent kinase 5 is mediated by GCN5. Biochem Bioph Res. Co, 447:121-127.

Lerin C, Rodgers JT, Kalume DE et al. (2006). GCN5 acetyltransferase complex controls glucose metabolism through transcriptional repression of PGC-1alpha. Cell Metab, 3: 429-438.

Kikuchi H, Kuribayashi F, Mimuro H et al. (2015). Lack of GCN5 remarkably enhances the resistance against prolonged endoplasmic reticulum stressinduced apoptosis through up-regulation of Bcl-2 gene expression. Biochem Bioph Res. Co, 463: 870-875.

Liu SL, Wang C, Jiang T et al. (2016). The Role of Cdk5 in Alzheimer's disease. Mol Neurobiol, 53:43284342 .

Avvakumov N, Cote J (2007). The MYST family of histone acetyltransferases and their intimate links to cancer. Oncogene, 26:5395-5407.

Tang Y, Luo J, Zhang W et al. (2006). Tip60-dependent acetylation of p53 modulates the decision between cellcycle arrest and apoptosis. Mol Cell, 24:827-839.

Vashishtha H, Ginsburg DS et al. (2016). Effects of Tip60 and Paclitaxel on breast and lung cancer. Clin Lab Sci, 29: 147-147.

Sabo SL, Lanier LM, Ikin AF et al. (1999). Regulation of $\beta$-amyloid secretion by Fe65, an amyloid protein precursor-binding protein. J Biol Chem, 274: 79527957.

Cao X, Südhof TC (2001). Transcriptively active complex of APP with Fe65 and histone acetyltransferase Tip60. Science, 293:115-120.

Lorbeck M, Pirooznia K, Sarthi J et al. (2011). Microarray analysis uncovers a role for Tip60 in nervous system function and general metabolism. PLoS ONE, 6: e18412.

Müller T, Schrötter A, Loosse C et al. (2013). A ternary complex consisting of AICD, FE65, and TIP60 down- 
regulates Stathmin1. BBA - Proteins Proteom, 1834:387-394.

[24] Sun Y, Sun J, Lungchukiet P et al. (2015). Fe65 suppresses breast cancer cell migration and invasion through Tip60 mediated cortactin acetylation. Sci Rep, 5: 11529 .

[25] Barbato C, Canu N, Zambrano N et al. (2005). Interaction of tau with Fe65 links tau to APP. Neurobiol Dis, 18: 399-408.

[26] Delatour B, Mercken L, El Hachimi KH et al. (2001). FE65 in Alzheimer's disease: Neuronal distribution and association with neurofibrillary tangles. Am J Pathol, 158:1585-1591.

[27] Xu S, Wilf R, Menon T et al. (2014). Epigenetic control of learning and memory in Drosophila by Tip60 HAT action. Genetics, 198: 1571-1586.

[28] Xu S, Elefant F (2015). Tip off the HAT- epigenetic control of learning and memory by Drosophila Tip60. Fly, 9: 22-28.

[29] Xu S, Panikker P, Iqbal S et al. (2016). Tip60 HAT action mediates environmental enrichment induced cognitive restoration. PLoS ONE, 0159623.

[30] Kitagishi Y, Nakanishi A, Ogura Y et al. (2014). Dietary regulation of PI3K/AKT/GSK-3 $\beta$ pathway in Alzheimer's disease. Alzheimers Res.Ther, 6: 35.

[31] Zhang Y, Ji G, Han S, Shao Z et al. (2018). Tip60 suppresses cholangiocarcinoma proliferation and metastasis via PI3k-AKT. Cell. Physiol Biochem, 50: 612-628.

[32] Kim JW, Jang SM, Kim CH et al. (2012). New molecular bridge between RelA/p65 and NF- $\kappa \mathrm{B}$ target genes via histone acetyltransferase TIP60 cofactor. J Biol Chem, 287: 7780-7791.

[33] Thomas T, Corcoran LM, Gugasyan R et al. (2006). Monocytic leukemia zinc finger protein is essential for the development of long-term reconstituting hematopoietic stem cells. Genes \& Dev, 20: 1175-1186.

[34] Lu P, Jones LL, Snyder EY et al. (2003). Neural stem cells constitutively secrete neurotrophic factors and promote extensive host axonal growth after spinal cord injury. Exp Neurol, 181: 115-129.

[35] Blurton-Jones M, Kitazawa M, Martinez-Coria H, et al. (2009). Hilda Martinez-Coria, Neural stem cells improve cognition via BDNF in a transgenic model of Alzheimer disease. PNAS, 106: 13594-13599.

[36] Millan F, Cho MT, Retterer K et al. (2016). Whole exome sequencing reveals de novo pathogenic variants in KAT6A as a cause of a neurodevelopmental disorder. Wiley Periodicals, 1791-1798.

[37] Perez-Campo FM., Costa G, Lie-a-Ling M et al. (2014). MOZ-mediated repression of p16INK4a is critical for the self-renewal of neural and hematopoietic stem cells. Stem cells, 32: 1591-1601.

[38] Perez-Gonzalez R, Antequera D, Perry G et al. (2011). Leptin induces proliferation of neuronal progenitors and neuroprotection in a mouse model of Alzheimer's disease. J Alzheimers Dis, 24: 17-25.

[39] Kitabayashi I, Aikawa Y, Yokoyama A et al. (2001). Fusion of MOZ and p300 histone acetyltransferases in acute monocytic leukemia with a $\mathrm{t}(8 ; 22)(\mathrm{p} 11 ; \mathrm{q} 13)$ chromosome translocation. Leukemia, 15: 89-94.

[40] Chaffanet M, Gressin L, Preudhomme C et al. (2000). MOZ is fused to p300 in an acute monocytic leukemia with t(8;22). Gene Chromosome Canc 28: 138-144.

[41] Kizuka Y, Kitazume S, Fujinawa R et al. (2015). An aberrant sugar modification of BACE1 blocks its lysosomal targeting in Alzheimer's disease. EMBO Mol. Med, 7: 175-189.

[42] Lu X, Deng Y, Yu D et al. (2014). Histone acetyltransferase p300 mediates histone acetylation of PS1 and BACE1 in a cellular model of Alzheimer's Disease. PLoS ONE, 9: e103067.

[43] Cook C, Stankowski JN, Carlomagno Y et al. (2014). Acetylation: a new key to unlock tau's role in neurodegeneration. Alzheimers Res Ther, 6: 29.

[44] Cook C, Carlomagno Y, Gendron TF et al. (2014). Acetylation of the KXGS motifs in tau is a critical determinant in modulation of tau aggregation and clearance. Hum Mol Genet, 23: 104-116.

[45] McGraw S, Morin G, Vigneault C et al. (2007). Investigation of MYST4 histone acetyltransferase and its involvement in mammalian gametogenesis. BMC Dev Biol, 7: 123.

[46] Kojima K, Kaneda K, Yoshida C et al. (2003). A novel fusion variant of the MORF and CBP genes detected in therapy - related myelodysplastic syndrome with t(10;16)(q22;p13). Brit J Haematol, 120:271-273.

[47] Valor LM, Viosca J, Lopez-Atalaya et al. (2013). Lysine Acetyltransferases CBP and p300 as Therapeutic Targets in Cognitive and Neurodegenerative Disorders. Curr. Pharm Design, 19:5051-5064.

[48] Cohen TJ, Guo JL, Hurtado DE et al. (2011). The acetylation of tau inhibits its function and promotes pathological tau aggregation. Nat Commun, 2: 252.

[49] Feng Y, Vlassis A, Roques C et al. (2016). BRPF3HBO1 regulates replication origin activation and histone H3K14 acetylation, EMBO J, 35:176-192.

[50] Chen Z, Zhou L, Wang L et al. (2018). HBO1 promotes cell proliferation in bladder cancer via activation of Wnt/ $\beta$-catenin signaling. Mol. Carcinogen, 57: 12-21.

[51] Zhang Z, Hartmann H, Do VM et al. (1998). Destabilization of $\beta$-catenin by mutations in presenilin1 potentiates neuronal apoptosis. Nature, 395:698-702.

[52] Killick R, Ribe EM, Shawi AR et al. (2014). Clusterin regulates $\beta$-amyloid toxicity via Dickopf-1-driven induction of the Wnt-PCP-JNK. Mol Psychiatr, 19: 8898.

[53] Mishima Y, Miyagi S, Saraya A et al. (2011). The Hbo1-Brd1/Brpf2 complex is responsible for global acetylation of $\mathrm{H} 3 \mathrm{~K} 14$ and required for fetal liver erythropoiesis. Blood, 118: 9.

[54] Han Z, Wu H, Kim S et al. (2018). Revealing the protein propionylation activity of the histone acetyltransferase MOF (males absent on the first). J Biol Chem, 293: 3410-3420. 
[55] Su J, Wang F, Cai Y et al. (2016). The Functional Analysis of Histone Acetyltransferase MOF in Tumorigenesis. Int. J. Mol. Sci, 17: 99.

[56] Cheignon C, Jones M, Atrián-Blasco et al. (2017). Identification of key structural features of the elusive $\mathrm{Cu}-\mathrm{A} \beta$ complex that generates ROS in Alzheimer's disease. Chem Sci, 8: 5107-5118.

[57] Angelova PR. Abramov AY (2018). Role of mitochondrial ROS in the brain: from physiology to neurodegeneration. FEBS Lett, 592: 692-702.

[58] Qiao W, Zhang W, Gai Y et al. (2014). The histone acetyltransferase MOF overexpression blunts cardiac hypertrophy by targeting ROS in mice. Biochem. Bioph Res Co, 448: 379-384.

[59] Zhong J, Ji L, Chen H et al. (2017). Acetylation of hMOF modulates $\mathrm{H} 4 \mathrm{~K} 16 \mathrm{ac}$ to regulate DNA repair genes in response to oxidative stress, Int J Biol Sci, 13: 923-934.
[60] Chatterjee A, Seyfferth J, Lucci J et al. (2016). MOF acetyl transferase regulates transcription and respiration in mitochondria. Cell, 167:722-738.

[61] Bordi M, Berg MJ, Mohan PS et al. (2016). Autophagy flux in CA1 neurons of Alzheimer hippocampus: Increased induction overburdens failing lysosomes to propel neuritic dystrophy. Autophagy, 12: 2467-2483.

[62] Goetzl EJ, Boxer A, Schwartz JB et al. (2015). Altered lysosomal proteins in neural-derived plasma exosomes in preclinical Alzheimer disease. Neurology, 7: 85.

[63] Sheikh BN, Bechtel Walz W, Lucci J et al. (2016). MOF maintains transcriptional programs regulating cellular stress response. Oncogene, 35:2698-2710.

[64] Li X, Li L, Pandey et al. (2012). The histone acetyltransferase MOF is a key regulator of the embryonic stem cell core transcriptional network. Stem Cell, 11: 163-178. 